\title{
ANTIBIOTICS ADMINISTRATION TO ENHANCE THE SUCCESS OF THERAPY IN SEPSIS PATIENTS
}

\author{
SAMUEL BUDI ${ }^{1 *}$, ZULLIES IKAWATI ${ }^{1}$, IWAN DWIPRAHASTO ${ }^{2}$, TITIK NURYASTUTI $^{3}$ \\ ${ }^{1}$ Department of Pharmacy, Gadjah Mada University, Indonesia. ${ }^{2}$ Department of Pharmacology and Toxicology, Gadjah Mada University, \\ Indonesia. ${ }^{3}$ Department of Clinical Microbiology, Gadjah Mada University, Indonesia. Email: sambud8011@yahoo.co.id
}

Received: 05 November 2016, Revised and Accepted: 24 January 2017

\section{ABSTRACT}

Objective: Sepsis is reported to cause $9.3 \%$ death from the total 250,000 patients in the United States. De-escalation of antibiotics is expected to increase the effectiveness of therapy, to decrease in the level of antibiotic resistance and to reduce mortality in sepsis patients. The purpose of this study was to describe the frequency and characteristics of de-escalation of antibiotics in patients with sepsis and its influence on the patient.

Methods: The study was conducted retrospectively with purposive sampling in hospitalized patients in a hospital in Yogyakarta, to the patients whose age are above 15 years, in the month of January to December 2015. Totally, 162 patients were included, in demographic, antibiotic regimens, duration of antibiotic treatment, de-escalation of antibiotics treatment, the culture results, mortality, and Length of Stay (LoS).

Result: The result showed that 116 patients were without de-escalation of antibiotics where the majority of cases is the absence of changes in the types of antibiotics that are given from the start of the therapy until the treatment is completed. The age of the most sepsis patients treated is about 46-60 years (36.4\%), LoS was 6-10 days highest (27.2\%), the number of patients received antibiotics for hospitalized majority 1-2 types of antibiotics (46.9\%), originally most of the patients' room are as many as those from emergency unit $63.6 \%$, clinical outcomes patients who were recovered as many as $46.3 \%$, the most comorbidities that frequently appear in sepsis patients is pneumonia is $616.6 \%$ cases. Patients who were examined culture is $29 \%$.

Conclusion: Giving antibiotics are appropriate and adequate to prevent the possibility of resistance and death, and the de-escalation strategy is a strategy that is safely used to treat patients with sepsis.

Keywords: Sepsis, Antibiotics, De-escalation of antibiotics, Culture.

(C) 2017 The Authors. Published by Innovare Academic Sciences Pvt Ltd. This is an open access article under the CC BY license (http://creativecommons. org/licenses/by/4. 0/) DOI: http://dx.doi.org/10.22159/ajpcr.2017.v10s2.19496

\section{INTRODUCTION}

Sepsis is a disease that often occurs and causes death in patients at several hospitals in the United States. It is then forwarded to the aim of trying to make a variety of ways or methods to lower the morbidity, mortality and appropriate technology approach. In recent years, many studies were then conducted by various strategic interventions to patients so getting the best clinical outcome [1].

The incidence of prevalence of patients with sepsis in the United States was found about 750,000 cases of sepsis in the United States. The average occurred in 3 cases out of 100,000 populations. When compared with others, acute myocardial infection occurred in a year of 900,000 cases while in acute stroke, it occurred in 700,000 cases. Simple data on average the largest cause of death in patients can be sorted as follows: Sepsis (29\%), acute myocardial infarction (25\%), and stroke $(23 \%)$. The average increase in patient mortality rate of severe sepsis and septic shock is $40 \%$ and $50 \%$ [1].

Prescribing antibiotics showed a major challenge to the physicians in their daily practice, particularly in making sure the design of clinical treatment. Thus, to think about it is how to deal with patients with severe sepsis therapy fast and precise antibiotic to decrease the morbidity and mortality [2]. However, the first phase should identify pathogenic bacteria and detect sensitivity to empirical antibiotics which would then be stopped or reduced the number of antibiotics and changed to a more narrow-spectrum antibiotics. This strategy is referred to as de-escalation therapy which turned out to be a right and appropriate theory to improve the precision of the appropriate therapy and lower the cost of treatment [2-4].
De-escalation of antibiotics is expected to increase the effectiveness of therapy. The decrease in the level of antibiotics resistance and to reduce the mortality in sepsis patients is high. The purpose of this study was to describe the frequency and characteristics of a de-escalation of antibiotics in patients with sepsis and its effect on patients.

Antibiotic therapy in sepsis should be given within no more than $1 \mathrm{hr}$ after the diagnosis of sepsis is made. Selection is based on the empirical antibiotics active against bacteria that cause and reach the source of the infection. Infections caused by MultiDrug-Resistant microbes should use traditional antibiotic combination. Antibiotics should be evaluated daily for the possibility of de-escalation. It should be carried out deescalation if the profile has been known sensitivity [5].

\section{MATERIALS AND METHODS}

Materials

The material used as the basis for making the data was from the patient record diagnosis of sepsis in the inpatient ward during JanuaryDecember 2015.

\section{Methods}

The researcher conducted a retrospective observational study based on medical records in inpatient ward of Dr. Sardjito hospital in Yogyakarta from January to December 2015. The study was conducted during March-August 2016. The population in this study is adult patients who are undergoing sepsis hospitalization in the period and where the research took place. Criteria for inclusion of research subjects includes adult patients with age $>15$ years and hospitalized in inpatient wards in the period and where the research took place. Research carried out by 
identifying a subject of research based on pre-defined criteria, recording the identity and reviewing their health status, observing the action culture specimens of patients, performing the treatment de-escalation local doctors and observing the clinical outcomes research subjects. Bacteria culture results obtained from data on culture examination germs in the patient's medical record. De-escalation of antibiotic treatment of patients was written and grouped by treatment criteria and influenced by multiple comorbidities who also suffered sepsis patients. The material used as the basis for making the data was from the patient record diagnosis of sepsis in the inpatient ward in JanuaryDecember 2015. Ethical committee approval in this study was issued by Medical and Health Research Ethics Committee (MHREC), Faculty of Medicine Universitas Gadjah Mada - Dr. Sardjito General Hospital.

\section{Inclusion criteria}

All inpatients, inpatient medicine, age >15 years diagnosed with sepsis are recorded in the medical record with clinical and laboratory inspection data in accordance with the criteria in the International Sepsis Definitions Conference.

\section{Exclusion criteria}

Patients whose medical record data are incomplete, lost, or damaged, patients who did not receive antibiotics and patients who go home at his own request before clinical action is completed.

\section{Data analysis}

This research data are the description without intervention so that the processing of data was done by grouping based on the problem. The calculation is made with the model number and percentage proportion. Each data are then analyzed carefully so that the data actually described the condition of the patient and the hospital.

\section{RESULTS}

This study was conducted using a sample of 162 patients consisted of 76 male patients and 86 female patients. From the research, it was found that the age of the patients whose age of 46-60 years with a range of $36.4 \%$ and the youngest is at the age of $76-91$ by $10 \%$. Length of stay (LoS) for the treatment of sepsis mostly for 6-10 days and antibiotics is used as much as 1-2 kinds of antibiotics given to patients (Table 1). From Table 2, it is showed that the comorbidities of the diagnosis of sepsis patients to the most turned out to be pneumonia [6].

In the management therapy of sepsis patients in internal medicine wards, it turns out the treatment in $29 \%$ of patients culture, in which the culture specimen sampling the vast majority were in sputum that is equal to $27.3 \%$ of the patients (Table 3 ). From the results of cultures, it showed that most bacteria were found in samples of patient specimens are Streptococcus sp. (Fig. 1). Several different bacteria found on culture results such as Staphyloccocus coagulase negative and Streptococcus viridans. They are often found in the causes of nosocomial infections originating from medical devices such as intravenous catheters.[ 5] Their Staphylococcus and S. viridans coagulase negative blood cultures cannot be set directly as a pathogenic organism for $85 \%$ of isolates.

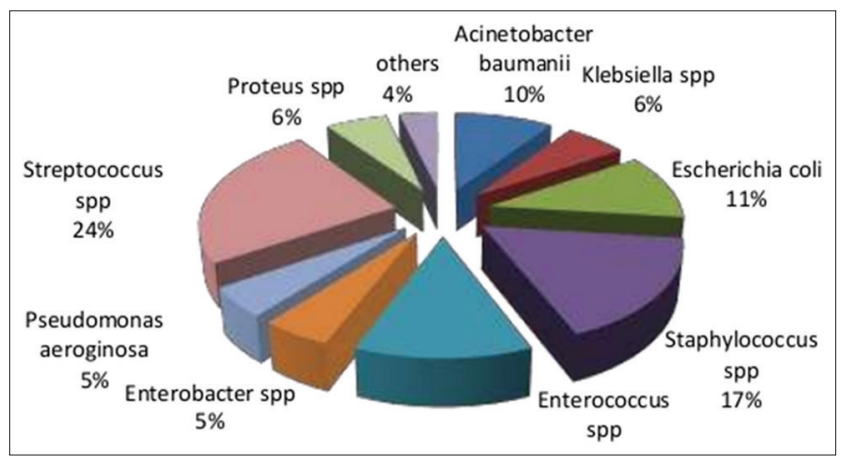

Fig. 1: Data germs in sepsis patients
Staphylococcus coagulase negative and $S$. viridans produced show contaminant organisms.[7]

Antibiotics are used to patients with sepsis in the majority class of cephalosporin that is as much as $42.58 \%$, quinolones at $17.48 \%$, as much as $10.77 \%$ carbapenem, aminoglycoside as much as $9.33 \%$, imidazoles as much as $8.85 \%$, others as much as $6,22 \%$, and macrolides

Table 1: Characteristic of adult being diagnosed sepsis patients in ward medicine hospital Dr. Sardjito 2015

\begin{tabular}{|c|c|c|}
\hline No & Characteristics & Frequency (\%) \\
\hline \multirow[t]{3}{*}{1} & Sex & \\
\hline & Man & $76(46.9)$ \\
\hline & Woman & $86(531)$ \\
\hline \multirow[t]{6}{*}{2} & Age (year) & \\
\hline & $15-30$ & $21(13)$ \\
\hline & $31-45$ & $32(19.8)$ \\
\hline & $46-60$ & $59(36.4)$ \\
\hline & $61-75$ & $40(24.7)$ \\
\hline & $76-91$ & $10(6.2)$ \\
\hline \multirow[t]{8}{*}{4} & Length of stay & \\
\hline & $0-5$ & $32(32)$ \\
\hline & $6-10$ & $44(27.2)$ \\
\hline & $11-15$ & $28(17.3)$ \\
\hline & $16-20$ & $27(16.7)$ \\
\hline & $21-25$ & $10(6.2)$ \\
\hline & $26-30$ & $9(5.6)$ \\
\hline & $>30$ & $12(7.4)$ \\
\hline \multirow[t]{7}{*}{5} & $\begin{array}{l}\text { Amount received antibiotics during } \\
\text { treatment }\end{array}$ & \\
\hline & 0 & $5(3.1)$ \\
\hline & $1-2$ & $76(46.9)$ \\
\hline & $3-4$ & $57(35.2)$ \\
\hline & $5-6$ & $14(8.6)$ \\
\hline & $7-8$ & $8(4.9)$ \\
\hline & $9-10$ & $2(1.2)$ \\
\hline
\end{tabular}

Table 2: Data accompanying disease in patients with sepsis

\begin{tabular}{lll}
\hline No & Accompanying disease & Total (\%) \\
\hline 1 & Pneumonia & $60(16.62)$ \\
2 & Hipoalbumin & $38(10.53)$ \\
3 & Diabetes mellitus & $32(8.86)$ \\
4 & Anemia & $27(7.48)$ \\
5 & Urinary tract infection & $26(7.20)$ \\
6 & CKD & $23(6.37)$ \\
7 & Hypertension & $22(6.09)$ \\
8 & Febrile neutropenia & $15(4.16)$ \\
9 & CHF & $14(3.88)$ \\
10 & Leukemia & $13(3.60)$ \\
\hline
\end{tabular}

CKD: Chronic kidney disease, CHF: Congestive heart failure

Tabel 3: The proportion of the culture of the specimen treatment

\begin{tabular}{lll}
\hline No & Tempat specimen kultur & Total (\%) \\
\hline 1 & Sputum & $18(27.3)$ \\
2 & Blood & $12(18.2)$ \\
3 & Urine & $10(15.2)$ \\
4 & Blood wound & $5(7.6)$ \\
5 & Pus & $11(16.7)$ \\
6 & Paring swab & $4(6.1)$ \\
7 & Swab nasofaring & $1(1.5)$ \\
8 & Faeces & $2(3)$ \\
9 & Pleura fluid & $1(1.5)$ \\
10 & Tissue & $1(1.5)$ \\
11 & Ulkus pedis & $1(1.5)$ \\
& Total & $66(100)$ \\
\hline
\end{tabular}


as much as $4.88 \%$. Other antibiotics in question are clindamycin, cotrimoxazole, rifampicin, ampicillin-sulbactam, tetracycline, etc (Fig. 2).

The treatment of de-escalation of antibiotics is one solution that is expected to be used in the treatment of patients with sepsis. From the research results, it was found that patients who receive treatment deescalation therapy were $28.4 \%$ and that no de-escalation was $71.6 \%$. In the act of de-escalation of antibiotics in patients with sepsis, it reduces the amount of antibiotics as much as $47.69 \%$, the replacement of dosage forms of antibiotics as much as $23.08 \%$, narrowing the $13.85 \%$ spectrum antibiotic therapy, antibiotic treatment discontinuation and dose reduction of antibiotics and $6.15 \%$ replacement generation of antibiotics to the smaller $3.08 \%$ (Table 4) [8-11]

The treatment of non de-escalation occurs in the majority of medical action in septic patients in the inpatient. When viewed from the non de-escalation it is measured that most antibiotics do not change the antibiotic combination of $45.73 \%$, then there is no change of antibiotic $29.20 \%$, did not change the spectrum antibiotic $24.09 \%$, the replacement of a single antibiotic combination to $3.65 \%$ (Table 4) [12].

\section{DISCUSSION}

De-escalation of antibiotics is the way of the treatment of pneumonia with two important features of the initial broad-spectrum antibiotics with high probability that cover all pathogens that may then within 48-72 hrs followed by the transfer of narrow-spectrum antibiotics by microbiological data that may include all germs (pathogens causative) [6,13]. An important role in de-escalation is the decisionmaking process by choosing a broad-spectrum therapy empirically, and policy discontinues antibiotic therapy when the results have been negative sputum culture microorganisms, and there are early signs of healing. $[9,10]$ De-escalation of antibiotics can also be interpreted as a strategy to align user needs adequate initial antibiotic therapy in highrisk patients to avoid unnecessary use of antibiotics that sparked the occurrence of resistance $[14,11]$.

If we look from Fig. 3 we can conclude that the handling of sepsis patients in hospitals still needs to be improved. This can be seen by the number of patients who died is quite high, especially patients died $>48$ hours. The amount was balanced with the number of patients who returned home the condition improved. Some way out to consider is the problem of treatment methods, especially on the appropriate antibiotics of both types, amount and time so that the outcome of patients can be better condition, especially reducing the number of patients who died to be improved or recovered [15].

From Table 5 we can see also that the implementation of antibiotic dealing in septic patients in hospitals has not been run in accordance with standard therapy. This may be due to several things such as the absence of robust microbiological or colonization data, conditions of disease complications and multi-resistance to antibiotics. Whereas in some research found that the implementation of antibiotic dehaling can decrease the incidence of antibiotic resistance, improving patient clinical outcome condition, and increasing cost effectiveness. By looking at some of the benefits of this antibiotic de-alteration then what may need to be done is to make a clear guidance to the physician that contains practical steps on how to do antibiotic dealing with a septic patient. $[15,16]$

\section{Broad-spectrum antibiotic treatment in empirical}

Interest de-escalation of antibiotics that have been mentioned above to minimize the resistance of germs to antibiotics and lower the cost of treatment. The broad-spectrum antibiotics are capable and effective against Gram-negative and Gram-positive and should be given immediately if there are signs of infection. This is done to prevent the high number of deaths, prevent dysfunction organ and reduce the number of hospitalizations due to inadequate early treatment. The broadspectrum antibiotics should also take into account the microbiological data and local patterns resistention. The principle strategy of deescalation is a strategy in a way that adequately antibiotics (potent) since the beginning of therapy to patients who have high-risk factors to avoid the use of antibiotics is not quite right. Antibiotics are inadequate and inaccurate that can lead to resistance. Another de-escalation

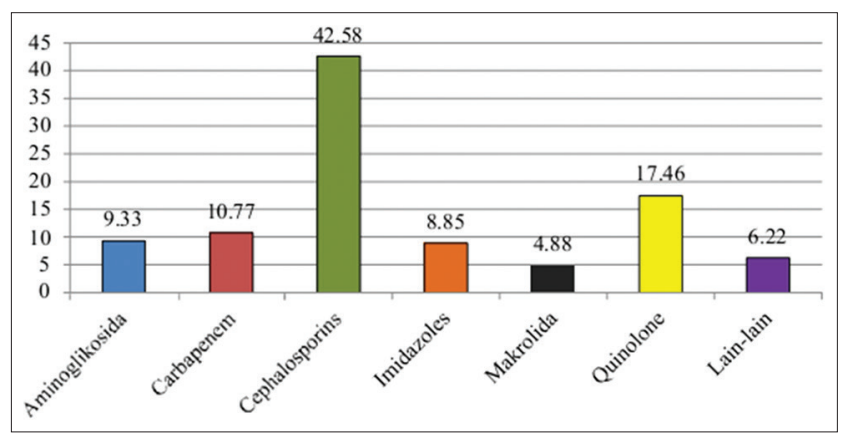

Fig. 2: Proportion of antibiotics used in sepsis patients

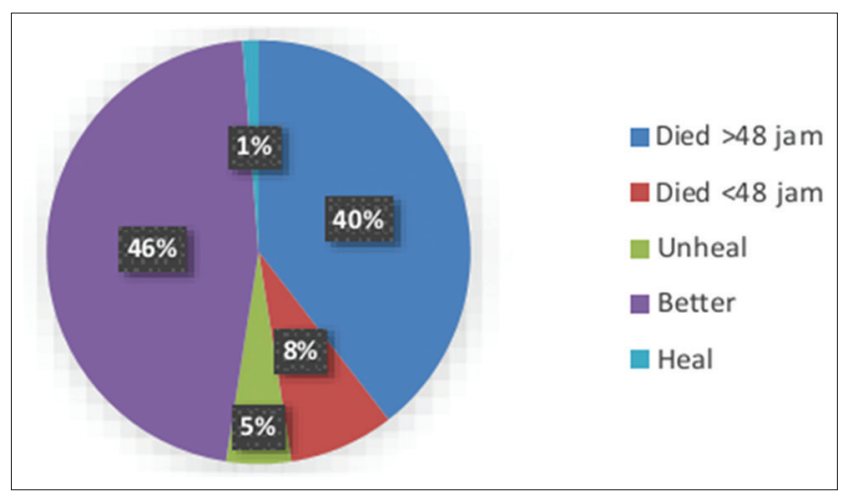

Fig. 3: Data clinical outcome of sepsis patients

Table 4: De-escalation antibiotics pattern sepsis patients

\begin{tabular}{|c|c|c|}
\hline Treatment of antibiotic therapy & Procedures therapy & Total (\%) \\
\hline \multirow[t]{7}{*}{ De-escalation } & Replacement of the dosage form antibiotic & $15(23.08)$ \\
\hline & Antibiotic dose reduction & $4(6.15)$ \\
\hline & Treatment discontinuation antibiotic & $4(6.15)$ \\
\hline & Narrow spectrum antibiotic therapy & $9(13.85)$ \\
\hline & Reducing the amount of antibiotics & $31(47.69)$ \\
\hline & Replacement antibiotic to smaller generation & $2(3.08)$ \\
\hline & Total & $65(100)$ \\
\hline \multirow{4}{*}{ Non de-escalation } & There is no change antibiotic & $40(29.2)$ \\
\hline & Replacement of antibiotic single to combination & $5(3.65)$ \\
\hline & Not changing the combination antibiotic & $59(45.73)$ \\
\hline & Total & $137(100)$ \\
\hline
\end{tabular}


Table 5: Proportion of de-escalation antibiotic treatment of sepsis patients

\begin{tabular}{lll}
\hline No & Treatment & Total (\%) \\
\hline 1 & De-escalation & $46(28.4)$ \\
2 & Nonde-escalation & $116(71.6)$ \\
\hline
\end{tabular}

strategy is to shorten the duration of administration of broad-spectrum antibiotics. Simplifying therapy becomes more narrow spectrum antibiotic and stop if cultures are negative bacteria as well as clinical improvement occurs. The strategy is done by providing initial therapy no more than 4 hrs since patients in the intensive care unit with broadspectrum antibiotics with high dose based on the pattern of bacteria microbiology local to lower mortality, prevent organ dysfunction and shorten the LoS and to optimize the therapeutic de-escalation to minimize resistance and save on the cost of treatment $[2,17]$.

\section{Giving antibiotics and dosage adequate accurately}

Hospital-acquired pneumonia patient's recovery can be achieved with the right combination of antibiotics and germs cause is still sensitive to these antibiotics as well as the provision of adequate antibiotic therapy. Appropriate antibiotic therapy and adequate in terms of the proper dosage (optimal dosage), the drug can reach the site of infection/ penetration into the tissue to cure the infection, antibiotics right way either intravenously or orally as well as in combination antibiotic therapy if possible. Giving a history of appropriate antibiotic therapy is one of the conditions of success managing severe sepsis and pneumonia. Determination of antibiotics should be based on knowledge of existing microorganisms, bacteria resistance patterns at the local site, the choice of drugs based on rational considerations $[6,18]$. Giving antibiotics is adequate since the beginning can improve survival rates.

\section{The combination therapy and monotherapy award}

Several strategies have been suggested in the unnecessary use of antibiotics as a trigger of the resistance of germs. The main objective of deescalation is trying to replace empirical treatment regimens combined into a monotherapy regimen to prevent resistance during treatment. At the American Thoracic Society-Infectious Diseases Society of America guidelines, it is said that patients with risk factors for tuberculosis (TB) germs suggested getting initial treatment with combination regimens that aim is to provide simple treatment that could include suspects multiple drug resistance (MDR) bacteria. Administration of drugs monotherapy in bacteria Pseudomonas aeruginosa and Enterobacter spp. is likely to cause drug resistance, for instance, given an only class of third generation cephalosporins [3]. Broad-spectrum antibiotic therapy should be added so that it gives the combination therapy to fight the germs that cause TB. Combined antibiotic combination therapy should be of a different class of therapeutic mechanisms to prevent antagonists. Combination therapy usually Gram-negative bacteria from the class of $\beta$-lactams, quinolones, or aminoglycosides. Although the combination of the quinolone class of better penetration than aminoglycosides and toxic effects to the kidneys is lower cure rates with drug combinations with aminoglycosides go on further [11].

Administration of drugs with monotherapy should be administered optimally. There are four criteria for combination therapy of antibiotics. First is when the combination provides synergy. The second is when the germs enter the category of weight and have not been identified. Third, if it can be proved that the combination therapy could delay the onset of resistance and the fourth case is a mixed infection of Grampositive bacteria, Gram-negative, and anaerobes. If these criteria are met and implemented correctly, it is little possible to the occurrence of MDR $[19,14,20]$.

\section{De-escalation narrow spectrum antibiotics}

There are several steps in the strategy of de-escalation of antibiotics: Starting with the administration of empirical antibiotic broad-spectrum with the aim of preventing the occurrence of therapy inadequate and inaccurate, change the treatment of a broad-spectrum into a narrow spectrum, reduction the duration of treatment and discontinue treatment based on the clinical response of patients and results microbiological cultures. De-escalation can be done if the culture results of data microbiology and germ profile have been out after a few days which is about 24-72 hrs to give a narrow spectrum antibiotic regimen or even stop the antibiotic if necessary. This strategy must be safe and appropriate in a change of combination therapy became monotherapy. This strategy should be based on microbiological data before it is treated. Negative cultures or bacteria were not found, and the clinical response of patients showed improvement after 2-3 days with treatment. The benefits of this strategy are that it can reduce cost, reduce mortality and morbidity due to inadequate antibiotic treatment $[6,14]$.

Therapeutic de-escalation can be performed on patients who demonstrated clinical response and more focused on the improvement of certain antibiotics as well as if there are no bacteria such as P. aeruginosa or Acinetobacter spp. Microbiological examination and cultures of microorganisms cultured specimens are still sensitive to the antibiotic lower orders.

\section{Shortening duration of antibiotics}

Shortening the duration or timing of empirical antibiotic therapy is shortened from a broad-spectrum approach de-escalation that can reduce the side effects of antibiotics and prevent the risk of germs against colonization and the growth of bacteria that are resistant to antibiotics as a result of antibiotic treatment is too long. Antibiotics briefly associated with a lower risk of antibiotic resistance compared to treatment with a time span of 14-21 days but if germs are not $P$. aeruginosa, the duration of antibiotic treatment could be shortened to 7-8 days [20,21].

\section{Rather therapy (switch therapy)}

The period of hospitalization should be shortened because it may reduce antibiotic resistance. The strategy by doing a parenteral antibiotic therapy to oral antibiotics is effective in the treatment of serious infections. In the execution of transfer therapy can be done by a stepdown therapy, sequential therapy, and switchover. Sequential therapy is shifting from intravenous to oral medication with the principle of adequate serum drug levels and maintained so that the drug levels are achieved intravenous and oral is the same as doxycycline, linezolid, and class quinolones such as levofloxacin and moxifloxacin. The transfer of oral therapy was associated with decreased serum drug levels compared with a step-down therapy intravenous known as third generation cephalosporin intravenously into oral amoxicillin or erythromycin. While the term switchover is the transfer of different types of drugs, but with the same drug levels, e.g. third generation cephalosporin intravenously into oral ciprofloxacin. Terms of the oral dosage must have pharmacokinetic properties and are able to achieve the target organ with levels above the minimum inhibitory concentration (MIC) so that it can eradicate infection-causing germs. One of the criteria pharmacokinetics of an oral antibiotic that can be used to control drug therapy was common ground potential or area under the curve with sediaan intravena or near $100 \%$, but the most important thing is the ability of the drug reaches the target organ with the same level or above the MIC. Some of the benefits obtained from the transfer of antibiotic therapy, these include the giving and administration of intravenous antibiotics reduce complications, improve patient compliance, reduce the incidence of pulmonary infection-causing germs resistance to antibiotics and can reduce the cost of care. Selection of antibiotics for this purpose can be done empirically and should be based on the pattern of resistance-causing germs and antibiotic resistance test data obtained locally [21].

\section{CONCLUSION}

Giving antibiotics are appropriate and adequate to prevent the possibility of resistance and death and the de-escalation strategy is a strategy that is safely used to treat patients with sepsis. 


\section{ACKNOWLEDGMENTS}

We would like to thank the staff of medical records, doctors and nurses in the department of Dr. Sardjito hospital, as well as the Faculty of Pharmacy, University of Gadjah Mada.

\section{REFERENCES}

1. Angus DC, Linde-Zwirble WT, Lidicker J, Clermont G, Carcillo J, Pinsky MR. Epidemiology of severe sepsis in the United States: Analysis of incidence, outcome, and associated costs of care. Crit Care Med 2001;29(7):1303-10.

2. Garnacho-Montero J, Garcia-Garmendia JL, Barrero-Almodovar A, Jimenez-Jimenez FJ, Perez-Paredes C, Ortiz-Leyba C. Impact of adequate empiric antibiotic therapy on the outcome of patients admitted to the intensive care unit with sepsis. Crit Care Med 2003;31(12):2742-51.

3. Kollef MH. The importance of antimicrobial resistance in hospitalacquires and ventilator associated pneumonia. Curr Anaesth Crit Care 2005;16(4):209-19.

4. Rello J, Vidaur L, Sandiumenge A, Rodríguez A, Gualis B, Boque C, et al. De-escalation therapy in ventilator-associated pneumonia. Crit Care Med 2004;32(11):2183-90.

5. Kakkilaya S. Rational Medicine: Rational Use of Antibiotics. Available from: http://www.rational medicine.org/antibiotics.htm. [Last cited on 2011 September 25]

6. Al-Nakeeb S, Elkholy A, Hafez SF, Basim H, Wasfy MO, ElShoubary W, et al. Procalcitonin testing has the potential to reduce unnecessary antibiotic use in patients with suspected lower respiratoy tract infections. Crit Care 2005;9(3):E5.

7. Saied T, Elkholy A, Hafez SF, Basim H, Wasfy MO, El-Shoubrary W. Antimicrobial resistance in pathogens causing nosocomial bloodstream infections in university hospitals in Egypt. Am J Infect Control 2011;39:61-5.

8. Rea-Neto A, Youssef NC, Tuche F, Brunkhorst F, Ranieri VM, Reinhart $\mathrm{K}$, et al. Diagnosis of ventilator-associated pneumonia. A systematic review of literature. Crit Care Med 2008;12:1186-97.

9. Lagamayo EN. Antimicrobial resistance in major pathogens of hospital-acquired pneumonia in Asian countries. Am J Infect Control 2008;36 4 Suppl: S101-8.
10. Niederman MS, Mandell LA, Anzueto A, Bass JB, Broughton WA, Campbell GD, et al. Guidelines for the management of adults with community-acquired pneumonia. Diagnosis, assessment of severity, antimicrobial therapy, and prevention. Am J Respir Crit Care Med 2001;163(7):1730-54.

11. Song JH; Asian Hospital Acquired Pneumonia Working Group. Treatment recommendations of hospital-acquired pneumonia in Asian countries: First consensus report by the Asian HAP Working Group. Am J Infect Control 2008;36 4 Suppl: S83-92.

12. Kollef MH. Optimizing antibiotic therapy in the intensive care unit setting. Crit Care 2001;5(4):189-95.

13. Tejerina E, Frutos-Vivar F, Restrepo MI, Anzueto A, Abroug F, Palizas F, et al. Incidence, risk factors, and outcome of ventilatorassociated pneumonia. J Crit Care 2006;21(1):56-65.

14. Niederman MS. The importance of de-escalating antimicrobial therapy in patients with ventilator-associated pneumonia. Semin Respir Crit Care Med 2006;27(1):45-50.

15. Masterton RG. Antibipotic de-escalation. Crit Care Clin 2011;27(1):149-62.

16. Licthenstern C, Nguyen TH, Schemmer P, Hoppe-Tichy T, Weigand MA. Efficacy of caspofungin in invasive candidiasis and candidemiade-escalation strategy. Mycoses 2008;51 Suppl 1:35-46.

17. Alvarez-Lerma F, Alvarez B, Luque P, Ruiz F, Dominguez-Roldan JM, Quintana E, et al. Empiric broad-spectrum antibiotic therapy of nosocomial pneumonia in the intensive care unit: A prospective observational study. Crit Care 2006;10(3):R78.

18. Chastre J, Fagon J. Ventilator-associated pneumonia: State of the art. Am J Respir Crit Care Med 2002; 165:867-93.

19. American Thoracic Society; Infectious Diseases Society of America. Guidelines for the management of adults with hospital-acquired, ventilator-associated, and healthcare-associated pneumonia. Am J Respir Crit Care Med 2005;171(4):388-416.

20. Paul M, Benuri-Silbiger I, Soares-Weiser K, Liebovici L. Beta lactam monotherapy versus beta lactam-aminoglycoside combinationtherapy for sepsis in immunocompetent patients: Systematic review and metaanalysis of randomised trials. BMJ 2004;328(7441):668

21. Nicolau DP. Pharmacodynamic optimization of beta-lactams in the patient care setting. Crit Care 2008;12 Suppl 4:S2. 